\title{
Slug to annular flow transition of microgravity two-phase flow
}

\author{
J.F. Zhao*, W.R. Hu \\ Institute of Mechanics, Chinese Academy of Sciences, Beijing, 100080, People's Republic of China
}

Received 17 January 1999; received in revised form 17 July 1999

\begin{abstract}
A new model is developed for predicting the transition from the slug to annular flow of adiabatic two-phase gas/liquid flow in microgravity $(\mu \mathrm{g})$ environment. This model is based on the analyses of the effects of the surface tension and the gas inertia in a sense of more physical approach. The drift-flux model is applied to determine the gas void fraction near the transition region. The new model is compared with previous models and experimental data, and the results show the improvement in explanation of the experimental results. (C) 2000 Elsevier Science Ltd. All rights reserved.
\end{abstract}

Keywords: Two-phase flow; Microgravity; Flow patterns; Slug-to-annular transition; Model

\section{Introduction}

Two-phase gas/liquid flow in microgravity $(\mu g)$ environment is an important subject for both academic and engineering purposes, and shows different features from that in normal gravity (1 $g$ ) environment (Dukler et al., 1988; Zhao, 1999). Only three distinct flow patterns exist typically in a $\mu g$ environment, i.e. bubble, slug and annular flow. Several experimental methods have been suggested to collect the data of flow patterns, for example, Reinarts (1993) used onecomponent vapor/liquid mixture and Zhao and Rezkallah (1993, 1995), Bousman (1995), and Rite and Rezkallah (1997) used two-component gas/liquid mixtures on board parabolic flight of airplane, while Lovell (1988) used equi-density, immiscible liquid/liquid systems and Mishima and Hibiki (1996) used miniscale capillary tubes on the ground. Models of flow

\footnotetext{
* Corresponding author.

0301-9322/00/\$ - see front matter (C) 2000 Elsevier Science Ltd. All rights reserved.

PII: S0301-9322(99)00088-9
} 
pattern transitions have also been suggested for $\mu g$ two-phase flow, such as the modification of $1 \mathrm{~g}$ models (Eastman et al., 1984; Karri and Mathur, 1988; Crowley et al., 1992), the linear stability analysis model (Carron and Best, 1994, 1996), the Weber number based model (Zhao and Rezkallah, 1993; Lee, 1987; Reinarts, 1995), the void fraction model (Dukler et al., 1988; Bousman, 1995; Colin et al., 1996), and the dimensional analysis model (Jayawardena et al., 1997).

The semi-empirical void fraction model was proposed firstly by Dukler et al. (1988). Using the drift-flux model (Zuber and Findlay, 1965) and assuming no local slip between the gas and liquid phases, the relationship between the gas and liquid superficial velocities for bubble and slug flow in $\mu g$ conditions is given as

$$
\frac{U_{\mathrm{SG}}}{U_{\mathrm{SG}}+U_{\mathrm{SL}}}=C_{0} \epsilon
$$

where $U_{\mathrm{SG}}$ and $U_{\mathrm{SL}}$ are respectively the gas and liquid superficial velocity, $\epsilon$ is the gas void fraction, and $C_{0}$ is an empirical parameter of gas distribution defined by the ratio of the gas averaged velocity to total superficial velocity of the two-phase mixture. For annular flow, the relationship of momentum balance between the gas and liquid phases gives

$$
\frac{\epsilon^{5 / 2}}{(1-\epsilon)^{2}}=\left(\frac{f_{\mathrm{I}}}{f_{\mathrm{L}}}\right)\left(\frac{\rho_{\mathrm{G}}}{\rho_{\mathrm{L}}}\right)\left(\frac{U_{\mathrm{SG}}}{U_{\mathrm{SL}}}\right)^{2},
$$

where $\rho_{\mathrm{G}}$ and $\rho_{\mathrm{L}}$ are, respectively, the gas and liquid densities, $f_{\mathrm{I}}$ and $f_{\mathrm{L}}$ are, respectively, the interfacial and liquid film friction factor. A modified Wallis model is suggested to determine $f_{\mathrm{I}}$, and the Blasius relation to $f_{\mathrm{L}}$. As the flow is expected to be a combination of slug and annular flows near the slug to annular transition region, Eqs. (1) and (2) can be used to solve the gas and liquid superficial velocities at the transition boundary.

In the Weber number based models, the surface tension and the gas inertia are considered as dominant factors. Slug or bubble flow will occur when the effect of the surface tension is greater than that of the gas inertia; otherwise, annular flow will occur. Lee (1987) suggested a semi-theoretical transition criterion as

$$
\rho_{\mathrm{G}} U_{\mathrm{G}}^{2}=\frac{2 \sigma}{R_{\mathrm{T}}}
$$

where $\sigma$ is the surface tension, $R_{\mathrm{T}}$ is the inside radius of the tube, and $U_{\mathrm{G}}$ is the gas averaged velocity equal to $U_{\mathrm{SG}} / \epsilon$. Eq. (3) can be rewritten in dimensionless form based on the gas Weber number as

$$
W e_{\mathrm{G}}=\frac{2 \rho_{\mathrm{G}} U_{\mathrm{G}}^{2} R_{\mathrm{T}}}{\sigma}=4
$$

Reinarts (1995) suggested that a factor $1 / 2$ ought to be added to the left-hand of Eq. (3), and that the radius upon which the surface tension acts must be the radius of the nose of the elongated gas bubble, and that the transition criterion must be written as 


$$
\frac{1}{2} \rho_{\mathrm{G}} U_{\mathrm{G}}^{2}=\frac{2 \sigma}{R_{\mathrm{B}}}
$$

where $R_{\mathrm{B}}$ is the radius of the nose of the elongated gas bubble. Eq. (2) was used with Eq. (5) to determine the void fraction and gas velocity for a given liquid velocity. An empirical relationship suggested by Wheeler (1992) was used to determine $f_{1}$ in the momentum balance relation.

Zhao and Rezkallah (1993) proposed a pure empirical model that the flow pattern transitions occur nearly at constant gas superficial Weber numbers defined by $W e_{\mathrm{SG}}=2 \rho_{\mathrm{G}} U_{\mathrm{SG}}^{2} R_{\mathrm{T}} / \sigma$. The authors defined a third flow pattern, frothy slug-annular flow, which is usually called slug-annular transition flow. The flow pattern map in terms of the gas and liquid superficial Weber number was obtained experimentally in $\mu g$ conditions for air/ water flows. It showed that the slug and bubble flow to frothy slug-annular flow transition and the frothy slug-annular flow to annular flow transition occur approximately at $W e_{\mathrm{SG}}=1$ and 20 , respectively. They then suggested that these values define the boundaries of the transition region.

Using the dimensional analysis, Jayawardena et al. (1997) suggested that the boundaries of flow pattern transitions depend on three dimensionless numbers, namely, the gas and liquid superficial Reynolds number and the liquid superficial Weber number, defined by $R e_{\mathrm{SG}}=2 \rho_{\mathrm{G}} U_{\mathrm{SG}} \mathrm{R}_{\mathrm{T}} / \mu_{\mathrm{G}}, \quad R e_{\mathrm{SL}}=2 \rho_{\mathrm{L}} U_{\mathrm{SL}} \mathrm{R}_{\mathrm{T}} / \mu_{\mathrm{L}} \quad$ and $W e_{S L}=2 \rho_{\mathrm{L}} U_{\mathrm{SL}}^{2} \mathrm{R}_{\mathrm{T}} / \sigma, \quad$ respectively. Experimental data were used to develop the flow pattern map, and the results showed that the slug to annular transition boundary might be expressed as

$$
\begin{aligned}
& \frac{R e_{\mathrm{SG}}}{R e_{\mathrm{SL}}}=K_{2} S u^{-2 / 3} \quad \text { for } S u>10^{6}, \\
& R e_{\mathrm{SG}}=K_{3} S u^{2} \quad \text { for } S u>10^{6},
\end{aligned}
$$

where the liquid Suratman number $S u_{\mathrm{L}}=R e_{\mathrm{SL}}^{2} / W e_{\mathrm{SL}}=2 \rho_{\mathrm{L}} \sigma R_{\mathrm{T}} / \mu_{\mathrm{L}}^{2} . K_{2}$ and $K_{3}$ are empirical parameters $\left(K_{2}=4641.6, K_{3}=2 \times 10^{-9}\right)$.

The present paper focuses on the modeling for the flow pattern transition from slug to annular flow in $\mu g$ environment, and is further restricted to the fully developed, one- and twocomponent, two-phase flows through adiabatic, circular cross-section tube of good wetted with the liquid phase.

\section{Idea of new model}

The new model of slug to annular transition in $\mu g$ conditions uses an approach similar to that of Lee (1987), Zhao and Rezkallah (1993) and Reinarts (1995), and assumes that the transition occurs if the impulsive force due to the gas inertia is sufficient to overcome the surface tension force which holds the elongated gas bubble whole and makes liquid bridge the tube. However, the analyses of the impulsive force and the relationship between the two forces are significantly changed.

The impulsive force due to the gas inertia acts upon the gas/liquid interface, which moves 
approximately at the same velocity as liquid bridge before the nose of the elongated gas bubble (Fig. 1). It is then better to characterize the impulsive force in a reference system moving together with the interface for a model to be more physically meaningful. In other words, the gas velocity ought to be expressed by the relative velocity in the impulsive force, and approximately written as $\left(U_{\mathrm{G}}-U_{\mathrm{L}}\right)$, where $U_{\mathrm{L}}$ is the liquid averaged velocity equal to $U_{\mathrm{SL}} /$ $(1-\epsilon)$. Therefore, the impulsive force may be characterized by $\rho_{\mathrm{G}} U_{\mathrm{SG}}\left(U_{\mathrm{G}}-U_{\mathrm{L}}\right)$, where the factor $\rho_{\mathrm{G}} U_{\mathrm{SG}}$ is the gas mass flow rate. The surface tension force acting upon the nose of the elongated gas bubble is characterized by $2 \sigma / R_{\mathrm{B}}$, as given by Reinarts (1995).

The surface tension and impulsive forces in the preceding expressions are characteristic values in orders of magnitude, but not the real local values. Thus, the transition condition may be expressed in order of magnitude as

$$
\rho_{\mathrm{G}} U_{\mathrm{SG}}\left(U_{\mathrm{G}}-U_{\mathrm{L}}\right)=O\left(\frac{2 \sigma}{R_{\mathrm{B}}}\right)
$$

Introducing a dimensionless factor $\kappa$, which is 1 in order of magnitude and may be determined empirically, the relation can be written as

$$
\frac{\rho_{\mathrm{G}} U_{\mathrm{SG}}\left(U_{\mathrm{G}}-U_{\mathrm{L}}\right) R_{\mathrm{B}}}{2 \sigma}=\kappa .
$$

The value of $R_{\mathrm{B}}$ may be determined by the assumptions similar to Reinarts (1995), that is: (1) the nose of the elongated gas bubble maintains the classic spherical shell shape for a Taylor bubble in slug flow near the transition region; (2) the nose radius can be approximated by the inside radius of the tube minus the film thickness if the gas and liquid phases were arranged in annular flow. Then the following relation is obtained

$$
R_{\mathrm{B}}=R_{\mathrm{T}} \epsilon^{1 / 2} \text {. }
$$

In order to determine the gas void fraction $\epsilon$ near the transition region, the drift-flux relation, Eq. (1), is applied in the present work, instead of the momentum balance relationship for annular flow used in both Weber number based models (Lee, 1987; Reinarts, 1995) and void fraction model (Duckler et al., 1988; Bousman, 1995). It is essentially the same thing because the flow near the transition region is expected to be a combination of slug and annular flow. Furthermore, the interfacial friction factor in the momentum balance relationship for annular flow is not known clearly, while considerable works have been done on the drift-flux model for slug flow in $1 \mathrm{~g}$ and $\mu \mathrm{g}$ conditions and the results show $C_{0} \approx 1.2$ (Fabre and Line,

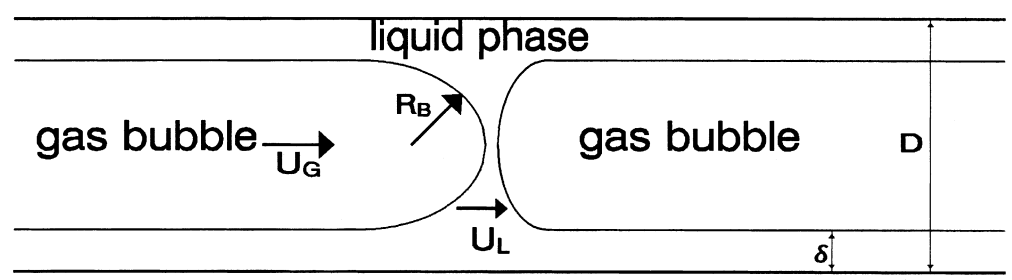

Fig. 1. Schema of the slug-to-annular transition. 
1992; Hewitt, 1996). The numerical simulations of Pan (Hewitt, 1996) give that $C_{0}$ is a constant near 1.16 in zero gravity conditions, and independent of mixture velocity. This value is consistent with the experimental result for bubble flows on board parabolic flight of airplane (Colin et al., 1996). Therefore, this value of $C_{0}$ is adopted in the present model for the slug to annular transition in $\mu g$ conditions. The value of another empirical parameter $\kappa$ in the new model can be determined by comparing the model with experimental data, which leads to $\kappa=0.8$. Thus, Eqs. (1) and (9) give a complete description of the new model.

It is beneficial to use the dimensionless rather than dimensional relations in the modeling for flow pattern transitions. Setting the characteristic velocity as $U_{\mathrm{C}}=\left(\sigma / 2 \rho_{\mathrm{G}} R_{\mathrm{T}}\right)^{1 / 2}$, and defining two dimensionless quantities

$$
\begin{aligned}
& W_{\mathrm{G}}=\frac{U_{\mathrm{SG}}}{U_{\mathrm{C}}}=\left(W e_{\mathrm{SG}}\right)^{1 / 2}, \\
& W_{\mathrm{L}}=\frac{U_{\mathrm{SL}}}{U_{\mathrm{C}}}=\left(\frac{\rho_{\mathrm{G}}}{\rho_{\mathrm{L}}}\right)^{1 / 2}\left(W e_{\mathrm{SL}}\right)^{1 / 2},
\end{aligned}
$$

the new model can be rewritten in the dimensionless form as

$$
\begin{aligned}
& W_{\mathrm{G}}=\left[\frac{4 \kappa C_{0} \epsilon^{1 / 2}(1-\epsilon)}{C_{0}-1}\right]^{1 / 2}, \\
& W_{\mathrm{L}}=\frac{1-C_{0} \epsilon}{C_{0} \epsilon} W_{\mathrm{G}},
\end{aligned}
$$

with $C_{0}=1.16$ and $\kappa=0.8$.

\section{Comparison and discussion}

The comparisons of the new model with the experiments in $\mu g$ conditions are shown in Fig. 2, which are plotted in the dimensional terms with the gas superficial velocity on the abscissa and the liquid superficial velocity on the ordinate. Fig. 2(a) plots the data of gas/liquid two-phase experiments with air/water mixture in a tube of $9.525 \mathrm{~mm}$ ID (Zhao and Rezkallah, 1993, 1995; Rite and Rezkallah, 1997), while Fig. 2(b) plots the data of R12 vapor/liquid mixture in a tube of $10.5 \mathrm{~mm}$ ID (Reinarts, 1993). The predictions of previous models proposed by Zhao and Rezkallah (1993), Bousman (1995), Reinarts (1995), and Jayawardena et al. (1997) are also plotted. All models predict slug flow in the left of the transition line and annular flow in the right.

The model of Zhao and Rezkallah (1993) predicts a transition region rather than a line. It is successful in separating the area of slug flow from that of annular flow for the air/water data. However, when compared with the R12 data, it predicts a transition region of too wide to involve some data points of both slug and annular flow. The models of Reinarts (1995) predict reasonably well at lower liquid superficial velocities, but underpredict the gas superficial 
velocity at higher liquid superficial velocities. The model of Bousman (1995) predicts an opposite tendency of the transition boundary. It predicts the air/water data with accuracy. However, for the R12 data, this model gives large under-prediction of the gas superficial velocity at lower liquid superficial velocities, and large over-prediction at higher liquid superficial velocities. The model of Jayawardena et al. (1997) predicts the same tendency of the transition boundary as that of Bousman (1995) for the air/water data where $S u<10^{6}$. However, the transition line predicted by this model is shifted up, and then the predicted area of annular flow involves some data points of slug flow. For the R12 data where $S u>10^{6}$, it predicts a constant gas superficial velocity, which is similar to that of Zhao and Rezkallah (1993), and successfully separates the area of slug flow from that of annular flow.

The prediction of the present model gives satisfaction for both the air/water and R12 data. It predicts a positive slope of the transition line for the air/water data, which agrees with the
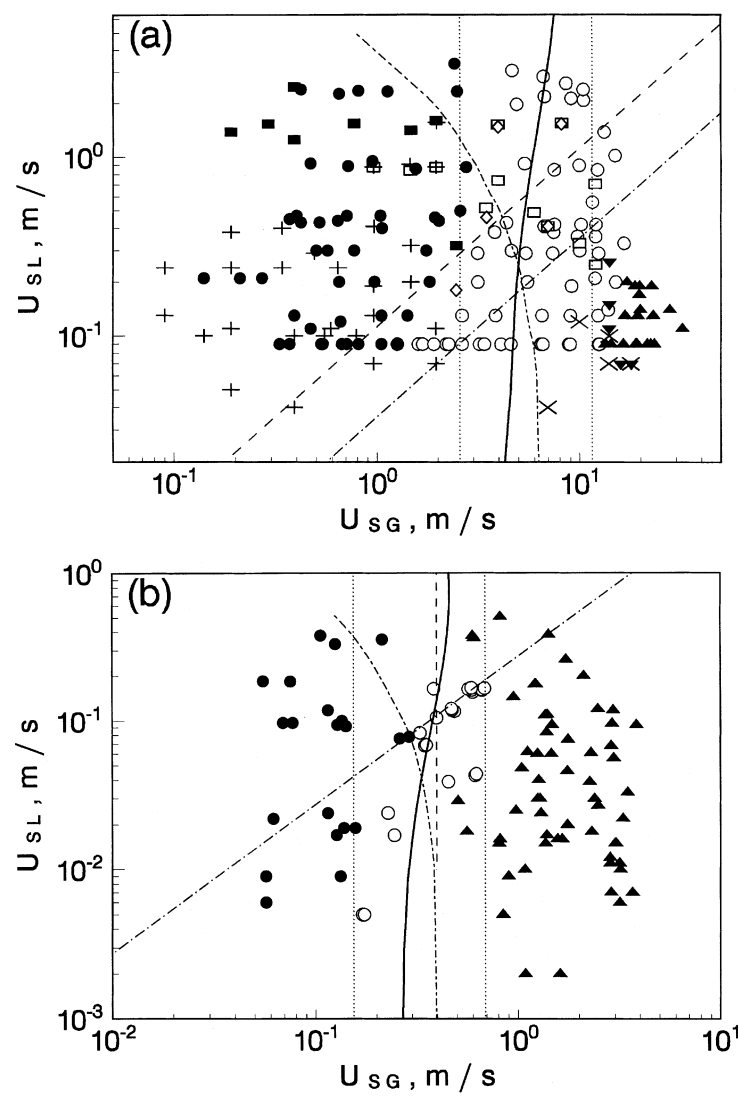

Fig. 2. Comparison of the present model with the experimental results and other models. Predictions: - the present model; . . . Zhao and Rezkallah (1993); — - Reinarts (1995); — - - Bousman (1995); - - Jayawardena et al. (1997). Experiments: (a) air/water flow in a tube of $9.525 \mathrm{~mm}$ ID. $\mathrm{slug}$, $\bigcirc$ slug-annular, $\boldsymbol{\Delta}$ annular (Zhao and Rezkallah, 1993); $\square$ slug, $\square$ slug-annular, $\nabla$ annular (Zhao and Rezkallah, 1995); + slug, $\diamond$ slug-annular, $\times$ annular (Rite and Rezkallah, 1997); (b) R12 vapor/liquid flow in a tube of $10.5 \mathrm{~mm}$ ID. slug, $\bigcirc$ slug-annular, $\boldsymbol{\Delta}$ annular (Reinarts, 1993). 
observation of Rezkallah (1996). For the R12 data, a positive slope is also observed at moderate liquid superficial velocities.

Furthermore, the present model can be plotted in a universal form by using the dimensionless quantities of $W_{\mathrm{G}}$ and $W_{\mathrm{L}}$ for different sets of experimental data. Fig. 3 plots the comparison of the present model with the gas/liquid two-phase flow experiments in $\mu g$ conditions (Bousman, 1995). Fig. 4 plots the comparison of the present model with the ground-based experiments of eqi-density, immiscible liquid/liquid systems (Lovell, 1988). Fig. 5 plots the comparison of the present model with the ground-based experiments of air/water capillary flow (Mishima and Hibiki, 1996). The slug-annular transition flow pattern data points are not plotted in Figs. 3 and 4 for clear and for the reason that identification of flow pattern near the transition boundary is a highly subjective matter. The predictions of other models are not plotted because they can not be plotted in this form for different experimental mediums and tube cross-section parameters, except the model of Zhao and Rezkallah (1993). It is clearly shown that the prediction of the present model is greatly satisfactory.

In general, the present model predicts accurately five sets of different experimental data covering a rather wide range of working fluids and tube diameters. It unifies the mechanism of the slug to annular transition of two-phase flow in both $\mu g$ experiments on board parabolic flight of airplane and simulating experiments on the ground, and then gives a unified classification of flow patterns based on the dimensionless quantities of $W_{\mathrm{G}}$ and $W_{\mathrm{L}}$. Therefore, it can be used to study flow patterns for any given two-phase system in $\mu g$ environments, even when there are no prior experimental data, and may be a useful approach for modeling of two-

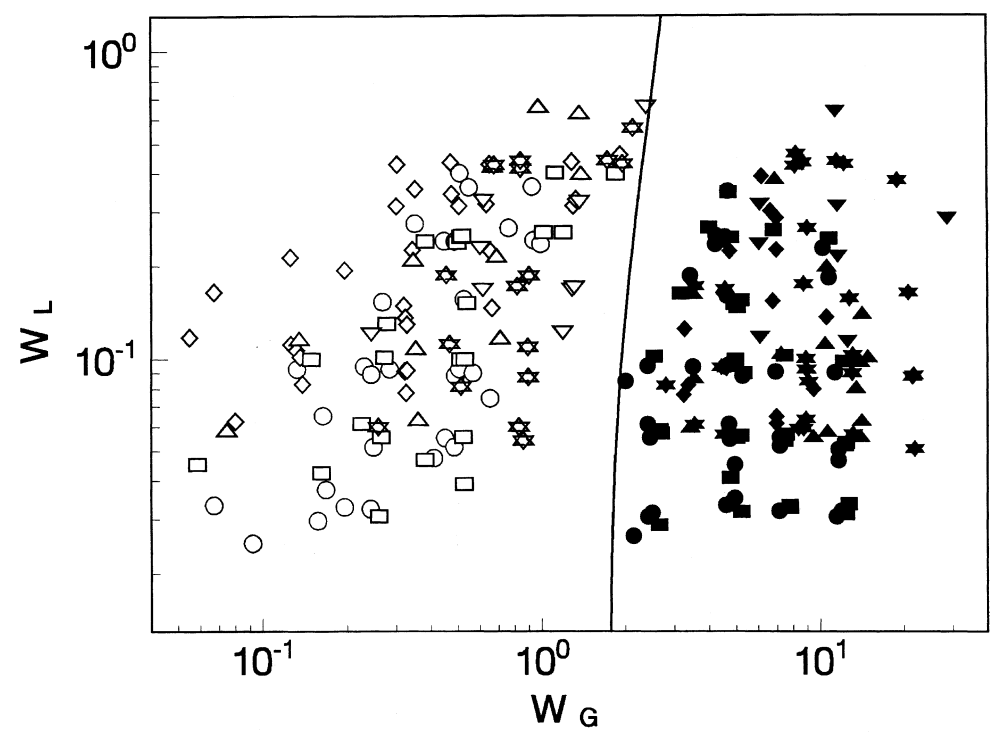

Fig. 3. Comparison of the present model with the gas/liquid experiments in low gravity conditions (Bousman, 1995). Open symbols: slug; closed symbols: annular. $\bigcirc$ air and water, $12.7 \mathrm{~mm} \mathrm{ID;} \diamond \diamond$ air and water, $25.4 \mathrm{~mm}$ ID; $\square$

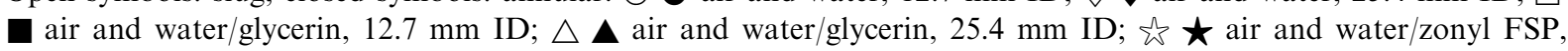
$12.7 \mathrm{~mm} \mathrm{ID} ; \nabla \nabla$ air and water/zonyl FSP, $25.4 \mathrm{~mm} \mathrm{ID}$; — prediction of the present model. 


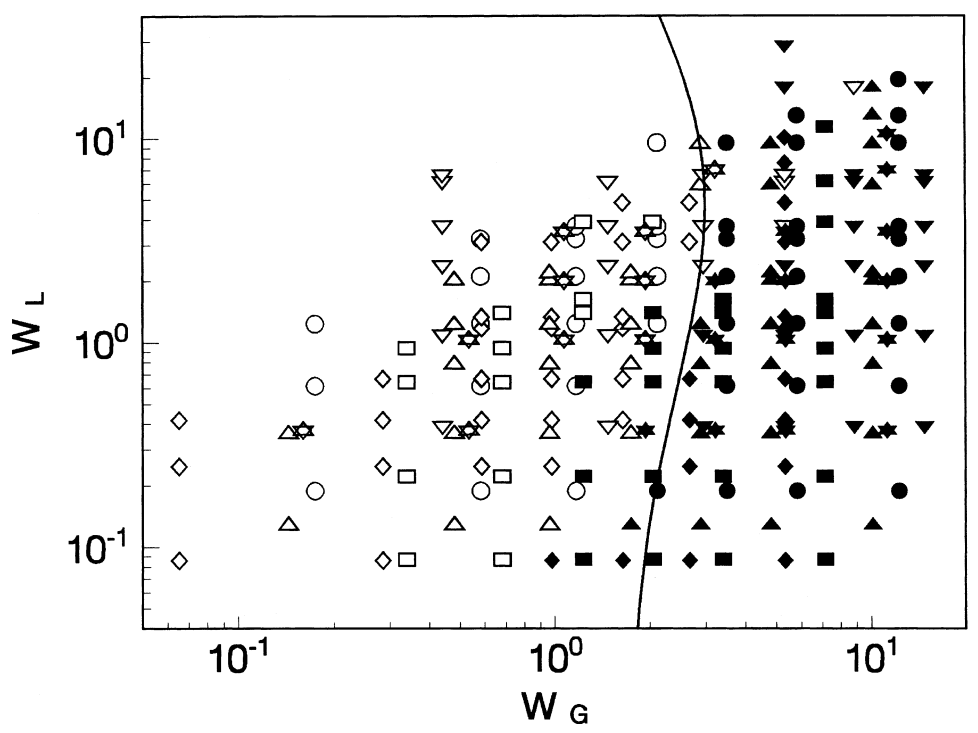

Fig. 4. Comparison of the present model with the liquid/liquid experiments on the ground (Lovell, 1988). Open symbols: slug; closed symbols: annular. $\bigcirc$ heavy mineral oil $/ \mathrm{CCl}_{4}, 25.4 \mathrm{~mm} \mathrm{ID} ; \diamond \diamond$ Dow Corning $200 \mathrm{fluid} /$ $\mathrm{CCl}_{4}, 8 \mathrm{~mm}$ ID; $\square$ Dow Corning 200 fluid/CCl $4,12.7 \mathrm{~mm} \mathrm{ID;} \triangle \boldsymbol{\Delta}$ Dow Corning $200 \mathrm{fluid} / \mathrm{CCl}_{4}, 25.4 \mathrm{~mm} \mathrm{ID;}$ is $\star$ kerosene $/ \mathrm{CCl}_{4}, 25.4 \mathrm{~mm} \mathrm{ID;} \nabla \nabla$ Dow Corning 200 fluid/CCl $\mathrm{Cl}_{4}$ with surfactant, $25.4 \mathrm{~mm}$ ID; - prediction of the present model.

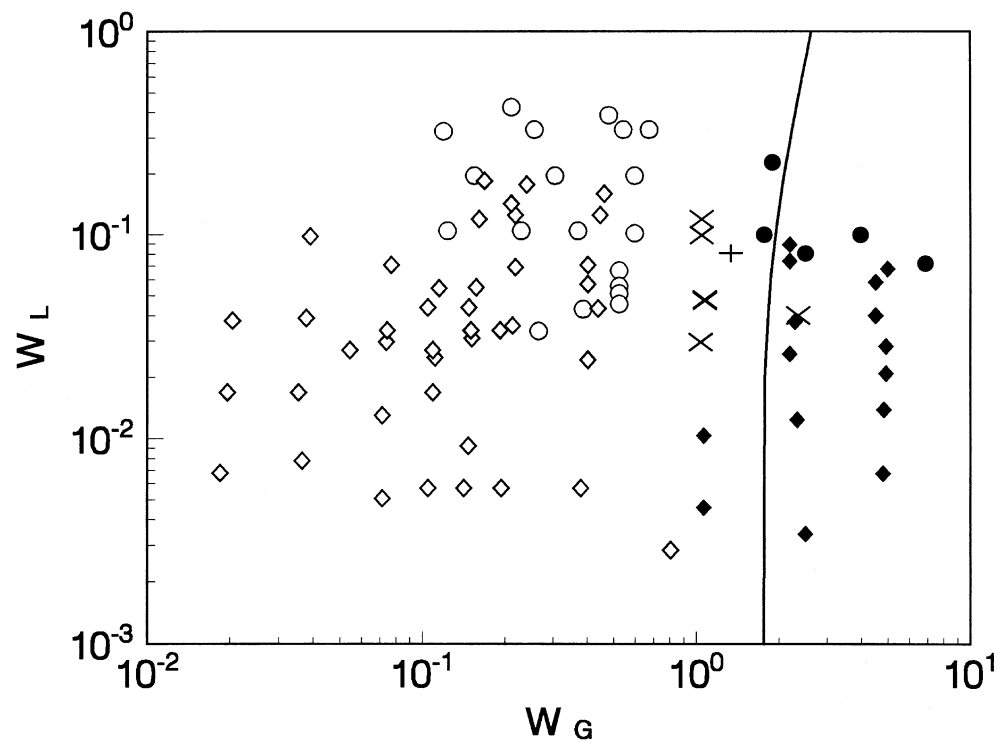

Fig. 5. Comparison of the present model with the capillary air/water experiments on the ground (Mishima and Hibiki, 1996). $\bigcirc$ slug, + slug-annular, annular (2.05 mm ID); $\diamond$ slug, $\times$ slug-annular, $\diamond$ annular (4.08 mm ID); - prediction of the present model. 
phase flow in such applications as two-phase ammonia thermal control systems (TCS) planned for the international space station.

\section{Conclusion}

A new model on the slug to annular transition has been developed for two-phase flows through adiabatic, circular cross-section tube of good wetted with liquid phase in $\mu g$ conditions. This model has been proved to be accurate over a rather wide range of working fluids, tube diameters, and experimental sorts. It offers an improvement of the accuracy in comparison with other models currently available in the literature. Furthermore, this new model is more physically based in some sense, and therefore has a much higher likelihood of being applicable outside of the calibration range.

However, there is more work to be done in the future. In fact, parameters $\kappa$ and $C_{0}$ are determined by the experiments, therefore, the present model is not completely founded on as a physical model. The model needs to be tested by more $\mu g$ two-phase flow experiments, particularly experiments of long period in orbital $\mu g$ conditions. More experimental and numerical investigations on slug propagation near the transition region in $\mu g$ conditions are also needed for determining accurately the value of $C_{0}$.

Furthermore, the approach presented in this paper can be applied to tubes of square or rectangular cross-section. Some modifications are needed, such as the value of $C_{0}$ and $\kappa$, and the relationship between $R_{\mathrm{B}}$ and the geometry parameters of cross-section, and so on.

\section{Acknowledgements}

This work is supported by grant 95-Yu-34 of the Ministry of Science and Technology and grant 19789201 of the National Natural Science Foundation of China. The authors would like to express their cordial thanks to Professor D.Y. Liu (Institute of Mechanics, CAS) for helpful discussions.

\section{References}

Bousman, W.S., 1995. Studies of two-phase gas-liquid flow in microgravity. PhD thesis, University of Houston.

Carron, I., Best, F.R., 1994. Gas-liquid annular flow under microgravity conditions: a temporal linear stability study. Int. J. Multiphase Flow 20, 1085-1093.

Carron, I., Best, F., 1996. Microgravity gas/liquid flow regime maps: can we compute them from first principles? In: Proceedings of AIChE Heat Transfer Symposium National Heat Transfer Conference, Houston, Texas.

Colin, C., Fabre, J., McQuillen, J., 1996. Bubble and slug flow at microgravity conditions: state of knowledge and open questions. Chem. Eng. Comm. 141/142, 155-173.

Crowley, C.J., Izenson, M.G., Barry, J., Martin, J.L., Ent, R.S., Valenzuela, J.A., 1992. Design manual for twophase components of spacecraft thermal management systems. PL-TR-92-3002.

Dukler, A.E., Fabre, J.A., McQuillen, J.B., Vernon, R., 1988. Gas-liquid flow at microgravity conditions: flow patterns and their transitions. Int. J. Multiphase Flow 14, 389-400. 
Eastman, R.E., Feldmanis, C.J., Haskin, W.L., Weaver, K.L., 1984. Two-phase fluid thermal transport for spacecraft. AFWAL-TR-84-3028.

Fabre, J., Line, A., 1992. Modeling of two-phase slug flow. Ann. Rev. Fluid Mech. 24, 21-46.

Hewitt, G.F., 1996. Multiphase flow: the gravity of the situation. In: Proceedings of Third Microgravity Fluid Physics Conference, Cleveland, Ohio.

Jayawardena, S.S., Balakotaiah, V., Witte, L.C., 1997. Flow pattern transition maps for microgravity two-phase flow. AIChE J. 43, 1637-1640.

Karri, S.B.R., Mathur, V.K., 1988. Two-phase flow pattern map predictions under microgravity. AIChE J. 34, 137139.

Lee, D., 1987. Thermohydraulic and flow regime analysis for condensing two-phase flow in a microgravity environment. PhD thesis, Texas A\&M University.

Lovell, T.W., 1988. Liquid-vapor flow regime transitions for spacecraft heat transfer loops. AFWAL-TR-88-3084.

Mishima, K., Hibiki, T., 1996. Some characteristics of air-water two-phase flow in small diameter vertical tubes. Int. J. Multiphase Flow 22, 703-712.

Reinarts, T.R., 1993. Adiabatic two phase flow regime data and modeling for zero and reduced (horizontal flow) acceleration fields. PhD thesis, Texas A\&M University.

Reinarts, T.R., 1995. Slug to annular flow regime transition modeling for two-phase flow in a zero gravity environment. In: Proceedings of 30th International Energy Conversion Engineering Conference, Orlando, FL.

Rezkallah, K.S., 1996. Weber number based flow-pattern maps for liquid-gas flows at microgravity. Int. J. Multiphase Flow 22, 1265-1270.

Rite, R.W., Rezkallah, K.S., 1997. Local and mean heat transfer coefficients in bubbly and slug flows under microgravity conditions. Int. J. Multiphase Flow 23, 37-54.

Wheeler, M.W., 1992. An experimental and analytical study of annular two phase pressure drop in a reduced acceleration field. MS thesis, Texas A\&M University.

Zhao, J.F., 1999. A review of two-phase gas-liquid flow patterns under microgravity conditions. Adv. In Mech. (Chinese), 29, 369-382.

Zhao, L., Rezkallah, K.S., 1993. Gas-liquid flow patterns at microgravity conditions. Int. J. Multiphase Flow 19, 751-763.

Zhao, L., Rezkallah, K.S., 1995. Pressure drop in gas-liquid flow at microgravity conditions. Int. J. Multiphase Flow 21, 837-849.

Zuber, N., Findlay, J.A., 1965. Average volumetric concentration in two-phase flow systems. J. Heat Transfer 87 , 453-468. 